\title{
Comparing the effect of induction therapy with or without antithymocyte globulin on renal allograft outcomes in live-donor kidney transplant recipients
}

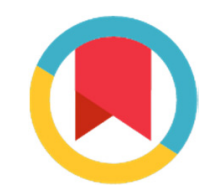

\author{
Bahareh Marghoob ${ }^{1}$, Neda Rahimian ${ }^{2}$, Yousef Ataiepour ${ }^{1}$, Marziyeh Mahdifarani ${ }^{1}$, Masoumeh Nejatifar ${ }^{3}$, Ali Kabir*4
}

Received: 12 Apr 2019

Published: 18 Dec 2019

\begin{abstract}
Background: This study aimed to investigate the effectiveness of antithymocyte globulin (ATG) in low-risk live-donor kidney transplant recipients (LDKTRs).

Methods: In this cohort study, 114 LDKTRs were analyzed in 2 groups of ATG induction therapy ( $\mathrm{n}=77)$ and control $(\mathrm{n}=37)$. In this study, $500 \mathrm{mg}$ pulse therapy with methylprednisolone was provided for both groups for 3 days. In addition, one $\mathrm{mg} / \mathrm{kg}$ of daily ATG was prescribed for 4 days in ATG induction group. Serum creatinine $(\mathrm{Cr})$ was measured at 3, 7, 30, 90, and 180 days after surgery and discharge day. Acute rejection (AR) was confirmed based on biopsy or rise in serum Cr by three-tenths from baseline if other causes had been ruled out. Survival analysis was used by Stata 14 and $p<0.05$ was considered significant.

Results: $\mathrm{Cr}$ changes were not significantly different between ATG induction therapy and control group in all follow-up periods (2.26 and 1.07 in ATG vs 2.26 and 1.03 in control group from the third day; $(p=0.999)$ to the sixth month $(p=0.735)$ ). There was no significant difference between the 2 study groups in AR incidence $(11.7 \%$ in ATG vs $10.8 \%$ in control group, $\mathrm{P}=0.890)$ and its time (9.6 in ATG vs 9.8 in control group, $\mathrm{p}=0.695$ ). Recipients factors were baseline $\mathrm{Cr}>10 \mathrm{mg} / \mathrm{dL}$ ( $\mathrm{p}=0.055$ ), blood group $\mathrm{AB}$ ( $\mathrm{p}=0.007$ ), no postoperative pulse therapy with methylprednisolone $(\mathrm{PM})(\mathrm{p}=0.005)$; and donors' factors were age $\leq 30$ years $(\mathrm{p}=0.022)$ and blood group $\mathrm{AB}(\mathrm{p}=0.006)$. Also, based on the log rank analysis, recipient-donor weight difference of 0 to $5 \mathrm{~kg}(\mathrm{p}=0.047)$ had a significant association with earlier AR. Exploring these effects simultaneously by Cox regression analysis showed only significant results for recipients' baseline $\mathrm{Cr}(\mathrm{p}=0.040)$ and postoperative therapy with $\mathrm{PM}(\mathrm{p}=0.014)$.

Conclusion: Both strategies of induction therapy had the same good results based on $\mathrm{Cr}$ decrease. Recipients' baseline $\mathrm{Cr}$ and postoperative therapy with PM were the predictors of survival time of the kidney (AR).
\end{abstract}

Keywords: Antithymocyte Globulin, Kidney Transplantation, Living Donors, Creatinine, Survival

Conflicts of Interest: None declared

Funding: The study was funded by grant number 28806 from Iran University of Medical Sciences

\section{*This work has been published under CC BY-NC-SA 1.0 license.}

Copyright $₫$ Iran University of Medical Sciences

Cite this article as: Marghoob B, Rahimian N, Ataiepour Y, Mahdifarani M, Nejatifar M, Kabir A. Comparing the effect of induction therapy with or without antithymocyte globulin on renal allograft outcomes in live-donor kidney transplant recipients. Med J Islam Repub Iran. 2019 (18 Dec);33:141. https://doi.org/10.47176/mjiri.33.141

\section{Introduction}

Induction therapy is an intense prophylactic therapy used at the time of transplantation to protect allograft against causes that have adverse effects on its survival (1). Acute rejection (AR) and delayed graft function (DGF) are the most important problems in any transplant procedure. Live-donor kidney transplant recipients (LDKTRs)

\section{Corresponding author: Dr Ali Kabir, kabir.a@iums.ac.ir}

1. HashemiNejad Hospital, Iran University of Medical Sciences, Tehran, Iran

2. Department of Internal Medicine, School of Medicine, Iran University of Medical Sciences, Tehran, Iran

3. Department of Epidemiology, School of Public Health and Safety, Shahid Beheshti University of Medical Sciences, Tehran, Iran

4. Minimally Invasive Surgery Research Center, Iran University of Medical Sciences, Tehran, Iran are assumed to be at lower risk of AR and DGF compared to deceased donor kidney transplant recipients (DDKTRs) (2). Eve n so, some studies have reported more rejection and a shorter rejection-free period among LDKTRs than DDKTRs, partially due to no induction therapy in this population $(3,4)$.

$\uparrow$ What is "already known” in this topic:

There is no consensus about the effectiveness of induction therapy with antithymocyte globulin (ATG) in live-donor kidney transplant recipients.

\section{$\rightarrow$ What this article adds:}

For live-donor kidney transplant recipients, induction regimen, including ATG, may not have additional advantages on graft function and prevention of acute rejection. 
In general, immunosuppressive regimens include induction, maintenance, and rescue agents. Induction agents include polyclonal antibodies (rabbit antithymocyte globulin (rATG)), interleukin-2 receptor antagonist (IL-2RA) (Basiliximab and Daclizumab), and Alemtuzumab and Rituximab, which are newer agents. The use of anti-CD3 monoclonal antibody (OKT3) has been limited because of its severe adverse side-effects. The maintenance therapy consists of calcineurin inhibitors (CNIs) (cyclosporine and Tacrolimus), antiproliferative agents (azathioprine and mycophenolate mofetil (MMF)), mammalian target of rapamycin inhibitors (mTOR-I) (Sirolimus and Everolimus), and corticosteroids. Belatacept (a costimulation blocker), sotrastaurin (a protein kinase $\mathrm{C}$ inhibitor), and tofacitinib (a Janus kinase (JAK)-3 inhibitor) are 3 newer agents in this group. Rescue agents, which include corticosteroids, polyclonal and monoclonal antibodies, immunoglobulins, rituximab, bortezomib, and eculizumab, are prescribed based on the type (cellular or acute antibodymediated) and severity (mild, moderate or severe) of renal rejection (5).

One of the induction agents whose effectiveness in the improvement of patient and allograft outcomes has been proven in DDKTRs and is routinely used in these patients is rATG- a polyclonal, T cell-depleting antibody (6). However, with respect to LDKTRs, this effect is unclear and there is no consensus about its routine use (1). Some studies have shown that ATG has a similar effect as basiliximab or alemtuzumab on AR in low-risk patients (including LDKTRs); however, it is more effective in high-risk patients (7). With regards to DGF, which has a lower rate in LDKTRs (up to 5\%-10\% of cases), it is controversial whether the induction therapy with ATG would help to decrease its incident and improve the next allograft long-term outcomes (8). To the best of our knowledge, less attention has been paid to study of the effect of induction therapy with ATG in renal transplantation in Iran (9, 10) and no study has been conducted on low-risk kidney transplant recipients. Thus, the aim of this study was to investigate the effectiveness of ATG as induction therapy in low-risk kidney transplant recipients in a referral transplantation center in Iran.

\section{Methods}

\section{Study setting}

This single-center retrospective cohort study was conducted in transplantation center of the Hasheminejad kidney center in Tehran between 2010 and 2014.

\section{Patients}

Patients who were diagnosed, for the first time, to be a candidate for renal transplantation from a living donor, with a negative screening for panel-reactive antibodies (absence of donor-specific antibodies: negative panel test) and no history of blood infusion were enrolled in the study. The exclusion criteria were high-risk patients for renal transplantation including cadaveric donor or second transplantation and more, multipara female patients, and positive panel test. With regards to sample size estimation, considering sigma $=0.47$ (the mean difference of the se- rum levels of creatinine between the 2 study groups at the first month after surgery), delta $=0.28$ (the standard deviation of the serum levels of creatinine for the both study groups at the first month after surgery), alpha $=0.05$ (the significant level), power $=80 \%$, and taking the exposed to unexposed cases ratio of 2 , using a power and sample size calculation software, the sample size in the ATG and control groups were estimated to be 70 and 34 , respectively (11).

\section{Immunosuppressive regimens}

Patients in ATG induction therapy received $500 \mathrm{mg}$ pulse therapy with methylprednisolone (PM) for 3 days with $1 \mathrm{mg} / \mathrm{kg}$ of daily ATG for 4 days. The control group only received $500 \mathrm{mg}$ PM for 3 days. For all patients, induction therapy was started from the night before surgery. The maintenance immunosuppressive for all patients was a triple regimen based on calcineurin inhibitor (cyclosporine with a dose of $3-5 \mathrm{mg} / \mathrm{kg}$ or tacrolimus with a dose of $0.1 \mathrm{mg} / \mathrm{kg}$ ) in combination with an antimetabolite (MMF) and prednisolone which was started from the third or fourth day after surgery.

\section{Preoperative evaluation}

In this phase, the basic information of patients, including demographic characteristics (age, sex), history of blood infusion, weight, and comorbidities (related to renal failure), were gathered using medical history records. The serum levels of creatinine and fasting blood sugar (FBS) were determined using blood sample. Comorbidities were categorized in 3 strata as below:

(1) Systemic diseases: Diabetes mellitus (DM), stone with or without DM, Wegner, hypertension with or without other diseases (DM, (FSGS), and IgA nephropathy; (2) kidney-related diseases: glomerulonephritis (GN), membranous glomerulonephritis (MGN), membranoproliferative glomerulonephritis (MPGN), polycystic kidney disease (PCKD), autosomal dominant polycystic kidney disease (ADPKD), FSGS, IgA nephropathy, urinary reflux, and proteinuria; and (3) Unknown.

In donors, the evaluation was based on demographic characteristics (age, sex), weight, and WBC cross-match.

\section{Postoperative evaluations and follow-up}

After surgery, the patients were examined for renal function, treatment need for AR or biopsy. Renal function was examined by assessing the serum levels of creatinine in all follow-ups at 3, 7, 30, 90, and 180 days after surgery and on the discharge day. The evaluation of the treatment need for AR was based on the rise in the serum levels of creatinine by as much as three-tenths compared to the baseline value or the results of the biopsy, if other causes had been ruled out.

\section{Statistical analysis}

For descriptive statistics, central and dispersion indices, such as mean and standard deviation (SD), were used for quantitative data, and number and percentage for qualitative data. In analytic statistics, with regards to the type (quantitative / qualitative) and role (independent / depend- 
ent) of the variables, parametric tests (independent t test, chi-squared, or Fisher's exact test, and logistic regression) and nonparametric tests (Mann-Whitney test) were used. Survival analysis for determining important variables for the time of graft rejection was implemented using logrank test and Cox proportional hazard regression. Data analysis was conducted using Stata14 (StataCorp LLC, USA), and $p<0.05$ was considered statistically significant. The significance level in log-rank test was less than 0.2. In case of nonsignificant association or difference, power was calculated to detect such relation by this sample size in each subgroup when they were compared.

\section{Results}

Data of 114 qualified patients were analyzed, of whom $58.77 \%(n=67)$ were male. The mean age of the patients was $43.49 \pm 12.82$ years and the mean weight was $66.89 \pm$ $14.06 \mathrm{~kg}$. There were no significant differences between the ATG induction therapy and control groups in these variables $(p=0.918, p=0.285$, and $p=0.580$, respectively). Moreover, the results of laboratory indices for creatinine and FBS showed that the baseline mean values for these indices were $7.60 \pm 3.12 \mathrm{mg} / \mathrm{dL}$ and $138.53 \pm 86.14 \mathrm{mg} / \mathrm{dL}$, with no significant differences between the 2 groups ( $p=0.111$ and $p=0.244$, respectively). Also, the blood groups $\mathrm{A}$ and $\mathrm{O}$ equally consisted the most patients' blood types ( $n=41,36.28 \%$ for each one). The distribution of blood group was not statistically different between the patients $(\mathrm{p}=0.098)$. Blood groups of patients and donors were the same in $59.43 \%(n=63)$ and $30.19 \%(n=32)$ of cases in ATG induction therapy and control groups, respectively $(p=0.510)$. Moreover, the distribution of comorbidities (relating to renal failure) of these groups was not significantly different $(\mathrm{p}=0.281)$. Overall, systemic diseases were the most frequently reported comorbidity $(n=56,49.12 \%)$, followed by kidney related diseases $(n=33,28.95 \%)$. Type of comorbidity was unknown for the remained patients $(n=25,21.93 \%)$. Details of basic characteristics of the patients are shown in Table 1. Most of donors were male $(\mathrm{n}=100,87.72 \%)$, with the mean age of $28.91 \pm 5.28$ years and the mean weight of $69.99 \pm 9.78$ $\mathrm{kg}$, with no significant differences between the 2 groups of induction therapy $(p=0.740, p=0.340$, and $p=0.529$ for sex, age and weight, respectively). Also, most donors had O $(n=42,40 \%)$ and $A(n=37,35.24 \%)$ blood types; and there was no significant difference between the study groups in this regard $(p=0.126)$ (Table 1$)$.

The changes of serum creatinine in all intervals of follow-up in the ATG induction therapy group were not significantly different from the control group. The mean values of these indices in days after kidney transplantation were $2.26 \pm 1.64 \mathrm{mg} / \mathrm{dL}$ at the third day, $1.67 \pm 1.41 \mathrm{mg} / \mathrm{dL}$ at the seventh day, $1.38 \pm 1.03 \mathrm{mg} / \mathrm{dL}$ at the time of discharge, $1.21 \pm 0.76 \mathrm{mg} / \mathrm{dL}$ at the first month, $1.13 \pm 0.63$ $\mathrm{mg} / \mathrm{dL}$ at the third month, and $1.06 \pm 0.52 \mathrm{mg} / \mathrm{dL}$ at the sixth month. Overall, $11.40 \%(n=13)$ of the patients had

Table 1. Basic characteristics of the patients and donors

\begin{tabular}{|c|c|c|c|c|c|}
\hline Characteristics & $\mathrm{ATG}^{\dagger}$ induction therapy & Control & Total & P-value & Power \\
\hline \multicolumn{6}{|l|}{ Patients properties } \\
\hline \multicolumn{6}{|l|}{ Demographic } \\
\hline \multicolumn{6}{|l|}{ Sex; N (\%) } \\
\hline Male & $45(58.44)$ & $22(59.45)$ & $67(58.77)$ & $0.918^{*}$ & 0.051 \\
\hline Female & $32(41.56)$ & $15(40.55)$ & $47(41.23)$ & & \\
\hline Age (Year); Mean \pm SD & $44.36 \pm 12.61$ & $41.52 \pm 13.26$ & $43.49 \pm 12.82$ & $0.285^{* *}$ & 0.180 \\
\hline Weight (kg); Mean \pm SD & $67.40 \pm 14.05$ & $65.83 \pm 14.23$ & $66.89 \pm 14.06$ & $0.580^{* *}$ & 0.188 \\
\hline \multicolumn{6}{|c|}{ Laboratory findings; Mean \pm SD } \\
\hline Serum Creatinine $(\mathrm{mg} / \mathrm{dl})^{\ddagger}$ & $7.27 \pm 2.91$ & $8.27 \pm 3.46$ & $7.60 \pm 3.12$ & $0.111^{* *}$ & 0.225 \\
\hline $\mathrm{FBS}^{\S}(\mathrm{mg} / \mathrm{dl})$ & $132.05 \pm 78.04$ & $152.38 \pm 101.13$ & $138.53 \pm 86.14$ & $0.244^{* *}$ & 0.228 \\
\hline \multicolumn{6}{|l|}{ Blood group; $\mathrm{N}(\%)$} \\
\hline A & $33(43.42)$ & $8(21.62)$ & $41(36.28)$ & $0.098^{*}$ & 0.544 \\
\hline B & $10(13.16)$ & $10(27.03)$ & $20(17.70)$ & & \\
\hline $\mathrm{AB}$ & $7(9.21)$ & $4(10.81)$ & $11(9.73)$ & & \\
\hline $\mathrm{O}$ & $26(34.21)$ & $15(40.54)$ & $41(36.28)$ & & \\
\hline \multicolumn{6}{|l|}{ Comorbidities; N(\%) } \\
\hline Systemic diseases & $38(49.35)$ & $18(48.64)$ & $56(49.12)$ & $0.281^{*}$ & 0.356 \\
\hline Kidney related diseases & $25(32.46)$ & $8(21.62)$ & $33(28.95)$ & & \\
\hline Unknown & $14(18.18)$ & $11(29.72)$ & $25(21.93)$ & & \\
\hline \multicolumn{6}{|l|}{ Donor properties } \\
\hline \multicolumn{6}{|l|}{ Sex; N(\%) } \\
\hline Male & $67(87.01)$ & $33(89.18)$ & $100(87.72)$ & $0.740^{*}$ & 0.062 \\
\hline Female & $10(12.98)$ & $4(10.81)$ & $14(12.28)$ & & \\
\hline Age (Year); Mean \pm SD & $29.27 \pm 5.08$ & $28.24 \pm 5.66$ & $28.91 \pm 5.28$ & $0.340^{* *}$ & 0.186 \\
\hline Weight (kg); Mean \pm SD & $69.57 \pm 10.14$ & $70.87 \pm 9.07$ & $69.99 \pm 9.78$ & $0.529^{* *}$ & 0.196 \\
\hline \multicolumn{6}{|l|}{ Blood group; $N(\%)$} \\
\hline A & $27(39.71)$ & $10(27.03)$ & $37(35.24)$ & $0.126^{*}$ & 0.497 \\
\hline B & $11(16.18)$ & $10(27.03)$ & $21(20)$ & & \\
\hline $\mathrm{AB}$ & $5(7.35)$ & & $5(4.76)$ & & \\
\hline $\mathrm{O}$ & $25(36.76)$ & $17(45.95)$ & $42(40)$ & & \\
\hline
\end{tabular}

$\dagger$ ATG: anti-thymocyte globulin; $\$ \mathrm{mg} / \mathrm{dl}$ : milligram per deciliter; $\S$ FBS: Fasting blood sugar; ${ }^{*}$ Comparison was done by Chi-square test; $* *$ Comparison was done by independent t-test. 
AR, with the mean time of $9.61 \pm 5.72$ days. There was no significant difference between the 2 groups of induction therapy in the incidence of $A R(n=9$ and $n=4$, in ATG induction therapy and control groups, respectively) $(p=0.890)$. Also, ATG induction therapy group had significantly 2.03 days of hospitalization more than the control group $(\mathrm{p}=0.022)$. The mean length of hospital stay (LoS) was $16.16 \pm 4.78$ and $14.13 \pm 3.05$ days, respectively. The result of comparing FBS at the time of discharge showed no significant difference between the 2 groups of induction therapy $(p=0.052)$, with the total mean of $139.31 \pm$ $57.64 \mathrm{mg} / \mathrm{dL}$ (Table 2). Most of the patients in both groups of induction therapy had no need for PM and ATG after surgery. Also, most of them used tacrolimus (Table 3).

The result of Kruskal-Wallis test showed that in the ATG group, there were significant differences between the 3 groups of baseline recipients' serum creatinine in levels of this marker at 3, 7, and 30 days after kidney transplantation and time of discharge $(p=0.006,0.022$, 0.017 , and 0.029 , respectively) (Table 4). This analysis had no significant results in the control group.

With regards to the effective factors in AR, the results of logistic regression showed that although LoS, the need for postoperative maintenance immunosuppressive therapy by PM, preoperative FBS, and recipient-donor weight difference had significant effects on $A R$ in univariate models, in multiple regression model, this effect remained significant only for the need to postoperative maintenance immunosuppressive therapy by PM and LoS. The need for postoperative maintenance immunosuppressive therapy by PM increases the risk of AR to 201.97 times $(p=0.001)$, and each day of hospitalization increases this indicator to 1.22 times $(p=0.037)$. Inversely, donor's weight had a significant effect only in multiple regression model in a way that each kilogram increase in donor's weight decreased the risk of AR by about $12 \%(p=0.023)$ (Table 5).

The results of log rank tests showed a significant relationship between time of AR and recipient factors, including baseline serum creatinine categories $(p=0.055)$, blood groups $(p=0.007)$, and the need for postoperative maintenance immunosuppressive therapy by PM $(p=0.005)$. Therefore, the mean time of AR was shorter in case of baseline serum creatinine above $10 \mathrm{mg} / \mathrm{dL}(4.33 \pm 3.05$ days), blood group AB (1 day), and no postoperative therapy by $\mathrm{PM}(6.75 \pm 3.57$ days $)$.

With regards to donor factors, age $(\mathrm{p}=0.022)$ and blood groups $(p=0.006)$ were significantly associated with the time of AR; donors' age $\leq 30$ and blood group AB were associated with shorter mean time of AR $(6.83 \pm 2.40$ and 1 days, respectively). Although the results of this test were not significant for the weight of patients or donors, it showed significant results in relation to categories of recipient-donor weight difference $(p=0.047)$ (Table 6). The mean time of AR in cases that this difference was 0 to 5 $\mathrm{kg}$ or 11 to $20 \mathrm{~kg}$ was shorter (4 and $8.37 \pm 4.62$ days, re-

\begin{tabular}{|c|c|c|c|c|c|}
\hline Variable $\dagger$ & ATG induction therapy & Control & Total & P-value & Power \\
\hline \multicolumn{6}{|l|}{ Serum Creatinine $(\mathrm{mg} / \mathrm{dl})^{\ddagger}$} \\
\hline 3 rd day & $2.26 \pm 1.66$ & $2.26 \pm 1.62$ & $2.26 \pm 1.64$ & $0.999^{*}$ & 0.192 \\
\hline 7 th day & $1.68 \pm 1.42$ & $1.64 \pm 1.41$ & $1.67 \pm 1.41$ & $0.882^{*}$ & 0.187 \\
\hline Discharge & $1.39 \pm 1.02$ & $1.36 \pm 1.07$ & $1.38 \pm 1.03$ & $0.915^{*}$ & 0.187 \\
\hline 1 st month & $1.27 \pm 0.72$ & $1.08 \pm 0.84$ & $1.21 \pm 0.76$ & $0.222^{*}$ & 0.162 \\
\hline 3rd month & $1.20 \pm 0.56$ & $0.99 \pm 0.75$ & $1.13 \pm 0.63$ & $0.100^{*}$ & 0.158 \\
\hline 6th month & $1.07 \pm 0.52$ & $1.03 \pm 0.52$ & $1.06 \pm 0.52$ & $0.735^{*}$ & 0.184 \\
\hline \multicolumn{6}{|l|}{ Acute Rejection } \\
\hline Positive $\mathrm{AR}^{\S}(\mathrm{N} ; \%)$ & $9(11.68)$ & $4(10.81)$ & $13(11.40)$ & $0.890^{* *}$ & 0.052 \\
\hline AR time (day) & $9.55 \pm 6.72$ & $9.75 \pm 3.20$ & $9.61 \pm 5.72$ & $0.695^{* * *}$ & 0.055 \\
\hline length of hospital stay (day) & $16.16 \pm 4.78$ & $14.13 \pm 3.05$ & $15.50 \pm 4.39$ & $0.022^{* * *}$ & \\
\hline $\mathrm{FBS}^{\dagger \dagger}$ (at the time of discharge) $(\mathrm{mg} / \mathrm{dl})$ & $132.49 \pm 56.06$ & $153.91 \pm 59.04$ & $139.31 \pm 57.64$ & $0.052^{* * *}$ & 0.492 \\
\hline \multicolumn{6}{|c|}{$\begin{array}{l}\dagger \text { Values are mean } \pm \mathrm{SD} \text {, otherwise mentioned; } \$ \mathrm{mg} / \mathrm{dl} \text { : milligram per deciliter; } \S \text { AR: acute rejection; } \dagger \dagger \text { FBS: fasting blood sugar; } * \text { Comparison was done by independe } \\
\text { t-test; ** Comparison was done by Chi-square test; *** Comparison was done by Mann-Whitney test. }\end{array}$} \\
\hline \multicolumn{6}{|c|}{ Table 3. Status of maintenance immunosuppressive therapy by study groups } \\
\hline Immunosuppression type & ATC & duction therapy & Control & P-value & Power \\
\hline Pulse therapy with methylprednisolone & & $4(5.19)$ & $2(5.40)$ & $0.637^{*}$ & 0.050 \\
\hline ATG & & $6(7.79)$ & $3(8.10)$ & $0.953^{* *}$ & 0.050 \\
\hline \multicolumn{6}{|l|}{ Maintenance therapy } \\
\hline Cyclosporine & & $4(5.19)$ & $4(10.81)$ & $0.272^{* *}$ & 0.051 \\
\hline Tacrolimus & & $3(94.80)$ & $33(89.18)$ & & \\
\hline
\end{tabular}

† ATG: anti-thymocyte globulin; *Comparison was done by Fisher's exact test; ${ }^{* *}$ Comparison was done by Chi-square test.

Table 4. Comparing changes in serum creatinine level $(\mathrm{mg} / \mathrm{dl}) \S$ in ATG induction therapy by categories of recipients' baseline serum creatinine levels ${ }^{\dagger}$

\begin{tabular}{|c|c|c|c|c|}
\hline \multirow[t]{2}{*}{ Time } & \multicolumn{3}{|c|}{ Baseline serum creatinine levels } & \multirow[t]{2}{*}{ P-value } \\
\hline & $<5$ & $5-9.9$ & $\geq 10$ & \\
\hline Number & 12 & 51 & 14 & \\
\hline 3rd day & $1.6 \pm 0.60^{\mathrm{a}^{* *}}$ & $2.41 \pm 1.58^{\mathrm{a}}$ & $3.94 \pm 2.49$ & $0.006^{*}$ \\
\hline 7 th day & $1.14 \pm 0.14^{\mathrm{b}}$ & $1.83 \pm 1.49^{\mathrm{bc}}$ & $2.30 \pm 1.48^{\mathrm{c}}$ & $0.022^{*}$ \\
\hline Discharge & $1.12 \pm 0.24^{\mathrm{d}}$ & $1.55 \pm 1.13^{\mathrm{de}}$ & $1.58 \pm 0.42^{\mathrm{e}}$ & $0.029^{*}$ \\
\hline 1st month & $1.10 \pm 0.18^{\mathrm{f}}$ & $1.39 \pm 0.70^{\mathrm{f}}$ & $1.58 \pm 0.51$ & $0.017^{*}$ \\
\hline 3rd month & $1.07 \pm 0.17$ & $1.29 \pm 0.49$ & $1.35 \pm 0.56$ & 0.103 \\
\hline 6th month & $1.08 \pm 0.16$ & $1.23 \pm 0.31$ & $1.25 \pm 0.42$ & 0.125 \\
\hline
\end{tabular}




\begin{tabular}{|c|c|c|c|c|}
\hline \multirow[t]{2}{*}{ Variable } & \multicolumn{2}{|c|}{ Simple regression } & \multicolumn{2}{|c|}{ Multiple regression } \\
\hline & $\mathrm{RR}^{\ddagger}(95 \% \mathrm{CI})^{\S}$ & P-value & $\mathrm{RR}(95 \% \mathrm{CI})$ & P-value \\
\hline Pulse therapy with methylprednisolone & $62.50(6.49,601.69)$ & $<0.001$ & $201.97(9.64,4229.66)$ & 0.001 \\
\hline length of hospital stay & $1.18(1.06,1.32)$ & 0.002 & $1.22(1.01,1.48)$ & 0.037 \\
\hline Preoperative $\mathrm{FBS}^{\dagger \dagger}$ & $1.006(1.0007,1.01)$ & 0.026 & $1(0.99,1.01)$ & 0.237 \\
\hline Donor weight & $0.95(0.89,1.01)$ & 0.112 & $0.88(0.80,0.98)$ & 0.023 \\
\hline \multicolumn{5}{|l|}{ Recipient-donor weight difference $(\mathrm{kg})$} \\
\hline $0-5$ & 1 & & 1 & \\
\hline $6-10$ & $2.38(0.20,28.13)$ & 0.491 & $0.31(0.006,14.92)$ & 0.555 \\
\hline $11-20$ & $8.69(1.008,74.99)$ & 0.049 & $7.90(0.55,113.51)$ & 0.128 \\
\hline$>20$ & $1.61(0.13,18.83)$ & 0.703 & $2.42(0.10,58.69)$ & 0.586 \\
\hline \multicolumn{5}{|c|}{$\dagger$ AR: Acute rejection; $\$$ RR: Relative risk; $\S$ CI: confidence interval; $\dagger \dagger$ FBS: fasting blood sugar; $\$$ kg: kilogram. } \\
\hline \multicolumn{5}{|c|}{ Table 6. The results of log rank in relation to risk factors for $\mathrm{AR}^{\dagger}$ after renal transplantation } \\
\hline Variable & Number & ents & Mean and $\mathrm{SD}^{+}$of time of $\mathrm{AR}$ & P-value \\
\hline \multicolumn{5}{|c|}{ Recipients' baseline serum creatinine levels $(\mathrm{mg} / \mathrm{dl})^{\S}$} \\
\hline $2-5$ & & & $12 \pm 1.41$ & 0.055 \\
\hline $5-9$ & & & $11 \pm 6.11$ & \\
\hline$\geq 10$ & & & $4.33 \pm 3.05$ & \\
\hline \multicolumn{5}{|l|}{ Recipients' blood groups } \\
\hline A & & & $10.14 \pm 6.59$ & 0.007 \\
\hline B & & & $11 \pm 4$ & \\
\hline $\mathrm{AB}$ & & & 1 & \\
\hline $\mathrm{O}$ & & & $10 \pm 4.24$ & \\
\hline \multicolumn{5}{|l|}{ Pulse therapy with methylprednisolone } \\
\hline Yes & & & $14.2 \pm 5.76$ & 0.005 \\
\hline No & & & $6.75 \pm 3.57$ & \\
\hline \multicolumn{5}{|l|}{ Donors' age groups (year) } \\
\hline$\leq 30$ & & & $6.83 \pm 2.40$ & 0.022 \\
\hline$>30$ & & & $12 \pm 6.80$ & \\
\hline \multicolumn{5}{|l|}{ Donor s' blood groups } \\
\hline A & & & $9.66 \pm 7.08$ & 0.006 \\
\hline B & & & $11 \pm 4$ & \\
\hline $\mathrm{AB}$ & & & 1 & \\
\hline $\mathrm{O}$ & & & $11 \pm 3.46$ & \\
\hline \multicolumn{5}{|l|}{ Recipient-donor's weight difference $(\mathrm{kg})^{\dagger \dagger}$} \\
\hline $0-5$ & & & 4 & 0.047 \\
\hline $6-10$ & & & $18 \pm 7.07$ & \\
\hline $11-20$ & & & $8.37 \pm 4.62$ & \\
\hline$>20$ & & & $9 \pm 2.82$ & \\
\hline
\end{tabular}

spectively) than differences above $20 \mathrm{~kg}$ or 6 to $10 \mathrm{~kg}$. Moreover, this analysis showed no significant result in the study groups. The mean time of AR was $9.55 \pm 6.72$ and 9.75 \pm 3.20 days in ATG induction therapy and control groups, respectively.

The Cox regression analysis, by adjusting for donor age, blood groups of recipients and donors, and recipientdonor's weight difference showed significant results only in recipients' baseline serum creatinine levels $(p=0.040)$ and in the need for postoperative maintenance immunosuppressive therapy by PM $(p=0.014)$. Thus, per one $\mathrm{mg} / \mathrm{dL}$ increase in baseline serum creatinine of a recipient, the risk of earlier AR will increase to 1.96 times $(95 \% \mathrm{CI}$ : $1.03,3.71)$, and no postoperative therapy by PM will increase this risk to 285.31 times (95\% CI: 3.10, 26191.22).

\section{Discussion}

This study showed that induction therapy with ATG had no additional advantages on graft function and prevention of AR compared to routine induction therapy by pulsed steroid. A similar finding was reported by Nga et al in a retrospective cohort study on 90 LDKTR. Based on that study, creatinine levels at discharge and 6 months after discharge were $1.22 \pm 0.4$ and $1.33 \pm 0.5 \mathrm{mg} / \mathrm{dL}$ in the thy- moglobulin group, $1.28 \pm 0.5$ and $1.34 \pm 0.7 \mathrm{mg} / \mathrm{dL}$ in patients who were prescribed basiliximab, and $1.59 \pm 0.7$ and $1.36 \pm 0.5 \mathrm{mg} / \mathrm{dL}$ in patients not receiving induction therapy, with no significant difference between the study groups (12).

Another study by Abou-Jaoude et al on 45 low-risk kidney transplant recipients of different types of donors (cadaveric, living- or emotionally related) receiving daclizumab $(n=10)$ or ATG-Fresenius (ATG-F) $(n=35)$ showed significant differences in creatinine levels at discharge $(1.23 \pm 0.11$ and $2.18 \pm 0.43 ; \mathrm{p}<0.001)$ and at 1 month (1.21 \pm 0.06 and $1.49 \pm 0.16 ; \mathrm{p}=0.005)$, respectively (11). This finding may be due to lack of adjustment on some important variations between the study groups (eg, donor type).

Another retrospective cohort study conducted by Cicora et al on effectiveness and safety of ATG-F ( $n=23)$ compared with rATG $(n=24)$, with respect to recipients of deceased or living donor kidney transplants, showed that in both groups, the levels of serum creatinine was not significantly different at 1 week and 1 and 3 months. Comparing the index at month 3 with the baseline level showed significant decreases in both groups, from $7.76 \pm 3.45$ to $1.57 \pm 0.47 \mathrm{mg} / \mathrm{dL}$ and $7.36 \pm 2.49$ to $1.44 \pm 0.71 \mathrm{md} / \mathrm{dL}$ in 
the rATG and ATG-F groups, respectively. The incidence of AR was $16 \%(\mathrm{n}=4)$ and $17 \%(\mathrm{n}=4)$ in the $\mathrm{rATG}$ and ATG-F groups, respectively, with no significant difference between them (1).

A study by Santos et al on 12944 adult kidney transplant recipients from 2003 to 2013, categorized by induction regimen, who received ATG $(\mathrm{n}=9120)$, alemtuzumab $(n=1687)$, and basiliximab $(n=2137)$ showed that the adjusted risk for one-year AR was significantly lower by $30 \%$ for ATG $(\mathrm{HR}=0.70)$ and $35 \%$ for alemtuzumab $(\mathrm{HR}=0.65)$ compared to basiliximab. The 5-year patient and graft survival rates were not significantly different between the induction agents. Moreover, the risk was lower in the case of steroid included in maintenance immunosuppression regimen $(\mathrm{HR}=0.80)(7)$.

Results of a systematic review and meta-analysis by Morgan et al showed that alemtuzumab and rATG reduce the risk of AR compared to IL-2 (13). A randomized controlled trial by Woodle et al on 103 LDKTR in 2 groups of early corticosteroid withdrawal and ATG induction to chronic corticosteroid therapy, showed no significant difference between the groups in creatinine levels at $1,3,6$, and 12 months posttransplantation. The mean creatinine at 12 months posttransplant was $1.3 \pm 0.5 \mathrm{mg} / \mathrm{dL}$ and $1.2 \pm 0.32 \mathrm{mg} / \mathrm{dL}$ in 2 groups, respectively (14). Another trial by Pilch et al that compared the efficacy and safety of rATG and IL-2 receptor antagonists (Basiliximab and Daclizumab) in combination with tacrolimus, MMF, and steroid on 200 patients ( $\mathrm{n}=98$ in the IL-2, and $\mathrm{n}=102$ in the rATG), showed no difference in the incidence of AR, renal function, and graft survival between the 2 groups (15).

In this study, based on the log rank analysis, it was found that among recipients factors, baseline serum creatinine above $10 \mathrm{md} / \mathrm{dL}$, blood group $\mathrm{AB}$, and no postoperative therapy by $\mathrm{PM}$, and among donors factors age $\leq 30$ years and blood group $\mathrm{AB}$, and recipient-donor weight difference 0 to $5 \mathrm{~kg}$ were the significant risk factors associated with incidence of AR in a shorter time. Also, induction therapy by ATG had no remarkable effect on time of AR. Exploring the effects of these risk factors in Cox regression analysis showed only recipients' baseline serum creatinine, and no postoperative therapy with PM were associated with earlier AR. In a study by Gaber et al on 2322 LDKTR from 49 US transplant centers receiving rATG induction, the patient, graft, and rejection-free survival were similar between patients receiving versus those not receiving steroids at discharge (16).

A study conducted by Senel et al on 158 LDKTR (14 of them were approved for DGF) showed that lower donor weight was a predictive factor in the incidence of DGF. Moreover, the recipient/donor weight ratio in DGF group was significantly 0.23 higher than no DGF group (1.26 and 1.03 , respectively; $\mathrm{p}<0.02$ ). They explained that this fact could be related to smaller nephron mass in this group (17).

A study by Redfield et al on 64024 LDKTR between 2000 and 2014 showed that compared to donors aged younger than 50 years, the donor age above 50 years increases the risk of DGF by 1.26 times (95\% CI: 1.12 , 1.42). In addition, recipient and donor BMI above 25 $\mathrm{kg} / \mathrm{m}^{2}$ increase the risk of DGF by $1.33(95 \%$ CI: 1.18 , $1.49)$ and 1.17 (95\% CI: $1.05,1.31)$ times, respectively (18). Results of the US Scientific Renal Transplant Registry from 1990 to 1998 showed that recipients of kidneys from donors older than 55 years increase the risk of DGF, a form of acute graft failure, by 2-fold. Based on generous evidence, it is acceptable to ignore the donors' age, diabetes, or hypertension, marginal or suboptimum grafts for transplantation if the pretransplantation kidney biopsy sample is of acceptable quality (19). This may explain the finding of the study since the donors' age had variations between 19 and 41 years and donors with high risk ages were not included in the study.

There were some limitations in the study including small sample size, nonprobability sampling, and retrospective design. Due to its retrospective design, it was not possible to access data on some important variables such as BMI of the donors and patients, HLA mismatch, length of renal failure from the first time of clinical detection, treatment history in relation to renal failure, histopathology of grafts, surgery time, type and duration of anesthesia, cold ischemia time, and complications after surgery. To handle the limitations of the study design and selection bias, the samples were restricted to recipients of living donors only and other types of donors were excluded. Also, to increase the power of the study, 2 cases were considered in ATG induction therapy per each case in the control group. To the best of our knowledge, the vast majority of published studies in examining the effectiveness of ATG have been conducted on recipients of deceased donors and less attention has been paid to low-risk recipients. In addition, most studies had retrospective designs $(1-3,6,12,20)$. Thus, further studies with more methodologically sound design should be conducted to cover all important variables including intermediates and confounders in assessing the induction effect of ATG on short and long allograft outcomes in LDKTR.

\section{Conclusion}

Induction therapy with ATG had no additional advantages on graft outcomes and prevention of AR compared to routine/common induction regimen in kidney recipients of living donors. Baseline serum creatinine and no postoperative therapy with PM were the major risk factors for earlier AR.

\section{Acknowledgements}

The authors would like to thank all the cases who participated in this study, the colleagues who supported this study, and the mentors and professors.

\section{Funding}

The study was funded by grant number 28806 from Iran University of Medical Sciences, Tehran, Iran.

\section{Ethical approval}

This study was conducted in accordance with the ethical standards of the ethics committee of Iran university of medical sciences with this ethics code number: IR.IUMS.REC1395-28806, and with the 2013 Helsinki 
declaration and its later amendments or comparable ethical standards.

\section{Informed consent}

A signed written informed consent was obtained from all individual participants involved in the study.

\section{Conflict of Interests}

The authors declare that they have no competing interests.

\section{References}

1. Cicora F, Mos F, Paz M, Roberti J. Clinical experience with thymoglobulin and antithymocyte globulin-Fresenius as induction therapy in renal transplant patients: a retrospective study. Exp Clin Transplant. 2013;11(5):418-22.

2. Hardinger KL, Schnitzler MA, Koch MJ, Labile E, Stirnemann PM, Miller B, et al. Thymoglobulin induction is safe and effective in livedonor renal transplantation: a single center experience. Transplantation. 2006;81(9):1285-9.

3. Campbell SB, Hothersall E, Preston J, Brown AM, Hawley CM, Wall $\mathrm{D}$, et al. Frequency and severity of acute rejection in live-versus cadaveric-donor renal transplants. Transplantation. 2003;76(10):1452-7.

4. Lim WH, Chang SH, Coates PT, McDonald SP. Parental donors in live-donor kidney transplantation associated with increased rejection rates and reduced glomerular filtration rates. Transplantation. 2007;84(8):972-80.

5. Kumar A, Shrestha B. Evolution of Immunosuppressive Agents in Renal Transplantation :An Updated Review. Int J Stem Cell Res Transplant. 2016;4(3):158-72.

6. Martinez-Mier G, Soto-Miranda E, Budar-Fernandez LF, MateuRivera LJ, Gomez-Diaz A, Trujillo-Martinez MF, et al. Thymoglobulin Induction Therapy in Deceased Donor Kidney Transplantation: Single-Center Experience in Mexico. Transplant Proc. 2016;48(2):596-9.

7. Santos AH, Jr., Casey MJ, Womer KL. Analysis of Risk Factors for Kidney Retransplant Outcomes Associated with Common Induction Regimens: A Study of over Twelve-Thousand Cases in the United States. J Transplant. 2017;2017:8132672.

8. Schenker P, Ozturk A, Vonend O, Krüger B, Jazra M, Wunsch A, et al. Single-dose thymoglobulin induction in living-donor renal transplantation. Ann Transplant. 2011;16(2):50-8.

9. Zeynali J, Ataipour Y. Clinical outcome of Induction therapies in patients undergoing renal transplantation. Tehran Uni Med J. 2015;73(6):425-30. (Persian)

10. Raeisi D, Sohrabi N, Omrani H, Ghanati H, Zare M, Nasir Kansestani A, et al. Comparison study of Antithymocyte globulin and Methylprednisolone as induction therapy in kidney transplant patients refer to Imam Reza hospital, Kermanshah. Clin Res Paramed Sci. 2013;2(1):9-16. (Persian)

11. Abou-Jaoude M, Ghantous I, Najm R, Afif C, Almawi W. Daclizumab versus anti-thymocyte globulin-fresenius as induction therapy for low-risk kidney transplant recipients. Transplant Proceed. 2003;35(7):2731-2

12. Nga HS, Garcia PD, Contti MM, Takase HM, Carvalho MFCD, Andrade LGMD. Different induction therapies for kidney transplantation with living donor. J Bras Nefrol. 2015;37(2):206-11.

13. Morgan RD, O'Callaghan JM, Knight SR, Morris PJ. Alemtuzumab Induction Therapy in Kidney Transplantation: A Systematic Review and Meta-Analysis. Transplantation. 2012;93(12):1179-88.

14. Woodle ES, Peddi VR, Tomlanovich S, Mulgaonkar S, Kuo PC. A prospective, randomized, multicenter study evaluating early corticosteroid withdrawal with Thymoglobulin in living-donor kidney transplantation. Clin Transplant. 2010; 24(1):73-83.

15. Pilch NA, Taber DJ, Moussa O, Thomas B, Denmark S, Meadows $\mathrm{HB}$, et al. Prospective randomized controlled trial of rabbit antithymocyte globulin compared with IL-2 receptor antagonist induction therapy in kidney transplantation. Ann Surg. 2014;259(5):888-93

16. Gaber AO, Matas AJ, Henry ML, Brennan DC, Stevens RB, Kapur
$\mathrm{S}$, et al. Antithymocyte globulin induction in living donor renal transplant recipients: final report of the TAILOR registry. Transplantation. 2012;94(4):331-7.

17. Senel F, Karakayali H, Moray G, Haberal M. Delayed graft function: predictive factors and impact on outcome in living-related kidney transplantations. Ren Fail. 1998;20(4):589-95.

18. Redfield RR, Scalea JR, Zens TJ, Muth B, Kaufman DB, Djamali $A$, et al. Predictors and outcomes of delayed graft function after living-donor kidney transplantation. Transplant Int. 2016;29(1):81-7.

19. Perico N, Cattaneo D, Sayegh MH, Remuzzi G. Delayed graft function in kidney transplantation. Lancet. 2004;364(9447):1814-27.

Patel SJ, Duhart Jr BT, Krauss AG, Moore LW, Egidi MF, Amiri HS, et al. Risk factors and consequences of delayed graft function in deceased donor renal transplant patients receiving antithymocyte globulin induction. Transplantation. 2008;86(2):313-20 